\title{
Risk Factors for Anastomotic Leakage after Rectal Cancer Resection and Reconstruction with Colorectostomy. A Retrospective Study with Bootstrap Analysis
}

\author{
Rene Warschkow, $\mathbf{M D}^{1,3}$, Thomas Steffen, $\mathbf{M D}^{1}$, Jutta Thierbach, $\mathbf{M D}^{2}$, Thomas Bruckner, $\mathbf{P h D}^{3}$, \\ Jochen Lange, $\mathrm{MD}^{1}$, and Ignazio Tarantino, $\mathrm{MD}^{1}$ \\ ${ }^{1}$ Department of Surgery, Kantonsspital, St. Gallen, St. Gallen, Switzerland; ${ }^{2}$ Regional Blood Transfusion Service of the \\ Swiss Red Cross, St. Gallen, St. Gallen, Switzerland; ${ }^{3}$ Institute of Medical Biometry and Informatics, Universität \\ Heidelberg, Heidelberg, Germany
}

\begin{abstract}
Background. This study was designed to apply modern statistical methods to evaluate risk factors for anastomotic leakage after rectal cancer resection in a retrospective cohort of patients who received a colorectostomy. Whereas a diverting stoma and tumor height are considered proven risk factors for anastomotic leakage, a lack of evidence about additional risk factors persists.

Methods. In a single-center study, 527 consecutive patients who received a colorectostomy after rectal cancer resection between 1991 and 2008 were retrospectively assessed. In addition to traditional uni- and multivariate regression, locally weighted scatterplot smoothing (LOWESS) regression and bootstrap analysis were applied to increase internal validity.

Results. Anastomotic leakage occurred in 70 patients (13.3\%; 95\% confidence interval (CI), 10.5-16.5\%) and mortality was $2.5 \%$ (95\% CI, 1.4-4.2\%). Diverting stoma (odds ratio (OR), 0.4; 95\% CI, 0.17-0.61) and tumor height (OR, 0.88; 95\% CI, 0.8-0.94) were proven to be protective. Neoadjuvant radiotherapy (OR, 2.15; 95\% CI, 1.58-4.24) and intraoperative blood loss (OR, 1.05; 95\% CI, 1.02-1.09) had a derogatory effect. Bootstrap analysis identified pre-existing vascular disease $(95.5 \%)$, more advanced UICC stage III or IV tumors $(95.7 \%$ or $91.5 \%$, respectively), and intraoperative $(96.1 \%)$ and postoperative $(99.4 \%)$ blood substitution as harmful. Both intraoperative
\end{abstract}

(C) Society of Surgical Oncology 2011

First Received: 4 November 2010;

Published Online: 6 April 2011

R. Warschkow, MD

e-mail: rene.warschkow@kssg.ch and postoperative blood substitution caused a dosedependent increase in risk.

Conclusions. Applying statistical resampling methods identified intraoperative blood loss, blood substitution, vascular disease, and advanced UICC stage as risk factors for anastomotic leakage. Greater distances between the tumor and the anal verge and performance of a diverting stoma were associated with a decreased risk of anastomotic leakage.

Anastomotic leakage after total mesorectal excision (TME) for rectal cancer is a major concern of visceral and colorectal surgeons, because it leads to significant morbidity and mortality and may negatively influence oncologic outcome. ${ }^{1,2}$ Various studies have evaluated a variety of risk factors for the development of anastomotic leakage. For many reasons, the results from these studies were confusing. ${ }^{3}$ The definition of anastomotic leakage varies and has been applied to heterogeneous patient groups. Risk factors, such as body mass index or tumor height, which are measured on continuous scales, are arbitrarily grouped. Case-control studies are stratified for potential risk factors, such as UICC stage. Thus, these factors cannot be evaluated. ${ }^{4,5}$ Prospective studies often include heterogeneous patient groups with malignant and nonmalignant disease or are focused on intraperitoneal anastomoses. ${ }^{6,7}$ Randomized, controlled studies (RCT) are limited because they evaluate only one specific risk factor, such as construction of a prophylactic diverting stoma or the effect of neoadjuvant radiotherapy.

Until now, most studies have evaluated risk factors in a methodical, stereotypic manner. Namely, after uni- and multivariate logistic analysis, an additional stepwise variable selection process is performed and the resulting 
variables are identified or dismissed as important risk factors. ${ }^{6,8}$ Applying newer statistical methods could help to close the gap between limited RCTs and the confusion about risk factors resulting from retrospective studies. In 1979, Efron introduced bootstrap methodology. ${ }^{9}$ The bootstrap method involves generating a number of resamples of an observed dataset. Each of these resamples has a size equal to the observed dataset and is obtained by random sampling with replacement from the original dataset. In each resample, a distinct statistical analysis is performed and finally summarized. ${ }^{10,11}$ Since the availability of more powerful computers, bootstrap and other computationally intensive resampling methods became very popular in statistical research and are helpful tools to gain valid model-based inferences. ${ }^{12}$ Unfortunately, methods like bootstrapping have not found their way into practice-oriented surgical statistical analysis. Another promising method is locally weighted scatterplot smoothing (LOWESS) regression. This method fits simple models to localized subsets of data point by point to build up a function. ${ }^{13}$

The purpose of this study was to combine conventional uni- and multivariate analysis with recent statistical methods to evaluate risk factors for anastomotic leakage after rectal cancer resection in a retrospective cohort of patients who received a colorectostomy.

\section{PATIENTS AND METHODS}

In a retrospective, single-institution, cohort study, 726 patients who underwent primary rectal cancer resection between February 1991 and August 2008 were identified through a computer search of the institutional database. After exclusion for various reasons (Fig. 1), 527 patients were included for further analysis.

726 Consecutive patients with resection of primary rectal cancer 1991-2008

\footnotetext{
Exclusion of 199 patients:

- Abdominoperineal resection $(n=73)$

- Coloanal or ileoanal anastomosis $(n=96)$

- Hartmann's procedure $(\mathrm{n}=3)$

- Local excision $(\mathrm{n}=26)$

- Intraoperative death $(\mathrm{n}=1)$
}

\section{7 patients eligible for analysis}

FIG. 1 Patient selection

\section{Operative Technique}

The technique for surgical dissection was previously described in detail. ${ }^{14}$ The type of reconstruction was determined by the surgeon depending on the tumor and anatomic circumstances. As a standardized procedure, anastomoses were performed after mobilization of the splenic flexure using a double-stapled technique. ${ }^{15}$ An air leak test was routinely performed by a transanal insufflation of air with the anastomosis immersed in saline solution.

\section{Data Collection and Definitions}

Data concerning the patients' demographics, comorbidity, operative details, postoperative mortality, morbidity, and histological results were gathered retrospectively from medical records. Tumor height, defined as the distance between the tumor and the anal verge, was determined from the results of rigid rectosigmoidoscopy, endorectal sonography, MRI scans, and colonoscopy and was considered in this ranked order. Operative time, blood loss, and intraoperative blood substitution was obtained from the surgical protocol. Postoperative blood substitutions were extracted from the patients' medical file and only considered if they occurred before the third postoperative day, i.e., before a diagnosis of anastomotic leakage. Intra- and postoperative blood substitutions were counted as $300 \mathrm{ml}$ per each bottle and were cross-checked at the local blood bank. A pre-existing vascular disease was considered present when coronary, cerebral, or peripheral arterial occlusive disease was mentioned in the anamnesis, or when the diagnosis was registered in the medical file. All patients had histologically proven adenocarcinoma of the rectum; two rectal cancers were found simultaneously in each of six patients. Anastomotic leakage as the primary outcome was defined as the presence of a pelvic abscess with proof of anastomotic leakage by rectal examination, sigmoidoscopy, extravasation of endoluminally administered water-soluble contrast upon radiography or computed tomography, or proof of anastomotic leakage upon reoperations. These examinations were performed in patients with suspected anastomotic leakage and corresponding clinical symptoms. Before closure of a loop ileostomy, a contrast study was routinely performed. Neoadjuvant radiotherapy was performed according to an interdisciplinary tumor board decision, especially in patients with $\mathrm{uT} 3$ or uN+ staging results. Surgical complications were classified according to the Clavien-Dindo classification. ${ }^{16,17}$

\section{Statistical Analysis and Authorization}

The $\mathrm{R}$ environment (http://www.r-project.org) was used for all statistical analyses. A two-sided $p$ value $<0.05$ was considered statistically significant. Continuous data are 
expressed as the mean \pm standard deviation. Additionally, interquartile range (IQR) was calculated for the timing of anastomotic leakage. The $95 \%$ confidence intervals (CI) of binominal proportions were estimated according to a modified Wilson method. ${ }^{18}$ Missing values (intraoperative blood loss, $n=4$; tumor height, $n=7$; operative time, $n=2$; American Society of Anesthesiologists [ASA] stage, $n=3$; and pre-existing vascular disease, $n=9$ ) were replaced with the multiple imputation method. For comparing proportions, Chi-square statistics were applied. For correlation analysis, Spearman's rank correlations were computed.

The risk set analyzed included age, body mass index, tumor height, operative time, and intraoperative blood loss as continuous variables. Intraoperative blood substitution, postoperative blood substitution before postoperative day 3 , gender, ASA classification, pre-existing vascular disease in anamnesis, UICC tumor stage, neoadjuvant radiotherapy, diverting stoma, educational status of the surgeon, and the type of reconstruction performed during the colorectostomy were treated as factorial variables. Conventional analysis of anastomotic leakage included univariate logistic regressions for each risk factor, a multivariate logistic regression with all risk factors ("full model"), and a backward variable selection procedure based on the Akaike's information criterion (AIC). For continuous risk factors, an additional LOWESS regression analysis with estimation of point-wise 95\% CI was applied. Finally, a bootstrap of the backward variable selection of potential influence factors for anastomotic leakage with 3,999 permutated samples containing 527 patients each was performed. The selection rate was used as an indicator of the importance of a factor and the fraction of odds ratios greater than unity as the direction of a factor's influence. ${ }^{12}$

The study was approved for retrospective data analysis by the Swiss Federal Expert Commission for Physician Confidentiality, and by the institutional ethical review board.

\section{RESULTS}

Perioperative morbidity occurred in 213 patients (40.4\%; 95\% CI, 36.3-44.7\%). Complications were classified as Dindo I in 46 patients (8.7\%), as Dindo II in 67 patients (12.7\%), as Dindo III in 81 patients $(12.7 \%)$, and as Dindo IV in 19 patients (3.6\%). Anastomotic leakage occurred in 70 patients (13.3\%; 95\% CI, 10.5-16.5\%). Diagnosis of anastomotic leakage was made between postoperative days 3 and 34, at a median of 8 days postoperatively (IQR, 6-13 days). In 33 patients (6.3\%; 95\% CI, 4.5-8.7\%), a reoperation was required, whereas in the remaining 37 patients $(7 \%$; 95\% CI, 5.1-9.5\%), a conservative therapy was performed. Perioperative mortality occurred in a total of 13 patients $(2.5 \%$; $95 \%$ CI,
$1.4-4.2 \%)$. Four patients in the leakage group $(5.7 \%$; $95 \%$ CI, $1.8-14.2 \%)$ and 9 patients without leakage (2\%; $95 \%$ CI, $1-3.8 \%$ ) died. The difference in perioperative mortality did not reach statistical significance $(p=0.06)$. Operations were performed a median of 28 (IQR, 21.5-42) days after the completion of neoadjuvant radiotherapy. Of 167 patients who received neoadjuvant radiotherapy, 145 $(87.5 \%)$ received 44 Gy or $45 \mathrm{~Gy}$, and $13(7.7 \%)$ received $25 \mathrm{~Gy}$.

\section{Conventional Uni- and Multivariate Analysis}

The characteristics of the study population and the results of the uni- and multivariate analyses for potential risk factors are listed in Table 1. In univariate analysis with simple logistic regression intraoperative blood loss $(p=0.001)$, intraoperative $(p=0.026)$ and postoperative blood substitution $(p=0.035)$ were significantly associated with an increased risk. The effect of blood substitution was dose-dependent. A shorter distance between the anastomosis and the anal verge $(p=0.092)$ and longer operative time $(p=0.057)$ were associated with an increased risk of anastomotic leakage. Multivariate analysis identified a shorter distance to the anal verge as the only significant risk factor $(p=0.048)$. A diverting stoma was associated with a decreased risk $(p=0.07)$ and neoadjuvant radiotherapy with an increased risk $(p=0.1)$. This result was confirmed in a mixed model with the year of surgery as a random intercept. According to the stepwise backward variable selection (Table 2), intraoperative blood loss was the only risk factor significantly associated with anastomotic leakage $(p=0.003)$. An increased distance between the tumor and the anal verge $(p=0.051)$ and performance of a diverting stoma $(p=0.051)$ were associated with a decreased risk but without reaching the significance level; postoperative blood substitution $(p=0.085)$ and neoadjuvant radiotherapy $(p=0.135)$ also did not reach statistical significance.

\section{LOWESS Regression}

Analysis of the association between continuous factors and the leakage rate using LOWESS-regression is shown in Fig. 2. Increasing tumor height correlated with a decreased risk of anastomotic leakage if the tumor was located $10 \mathrm{~cm}$ or more from the anal verge. Longer operative time led to a proportionally increased risk. For blood loss between $700 \mathrm{ml}$ and $1500 \mathrm{ml}$, no change in the risk of leakage was observed. Below and over these limits, a decrease and increase, respectively, in risk occurred. In patients with a body mass index exceeding $25 \mathrm{~kg} / \mathrm{m}^{2}$, an increased risk for leakage was observed. Age had no relevant correlation. The effect of covariates on the leakage rate, adjusted for 


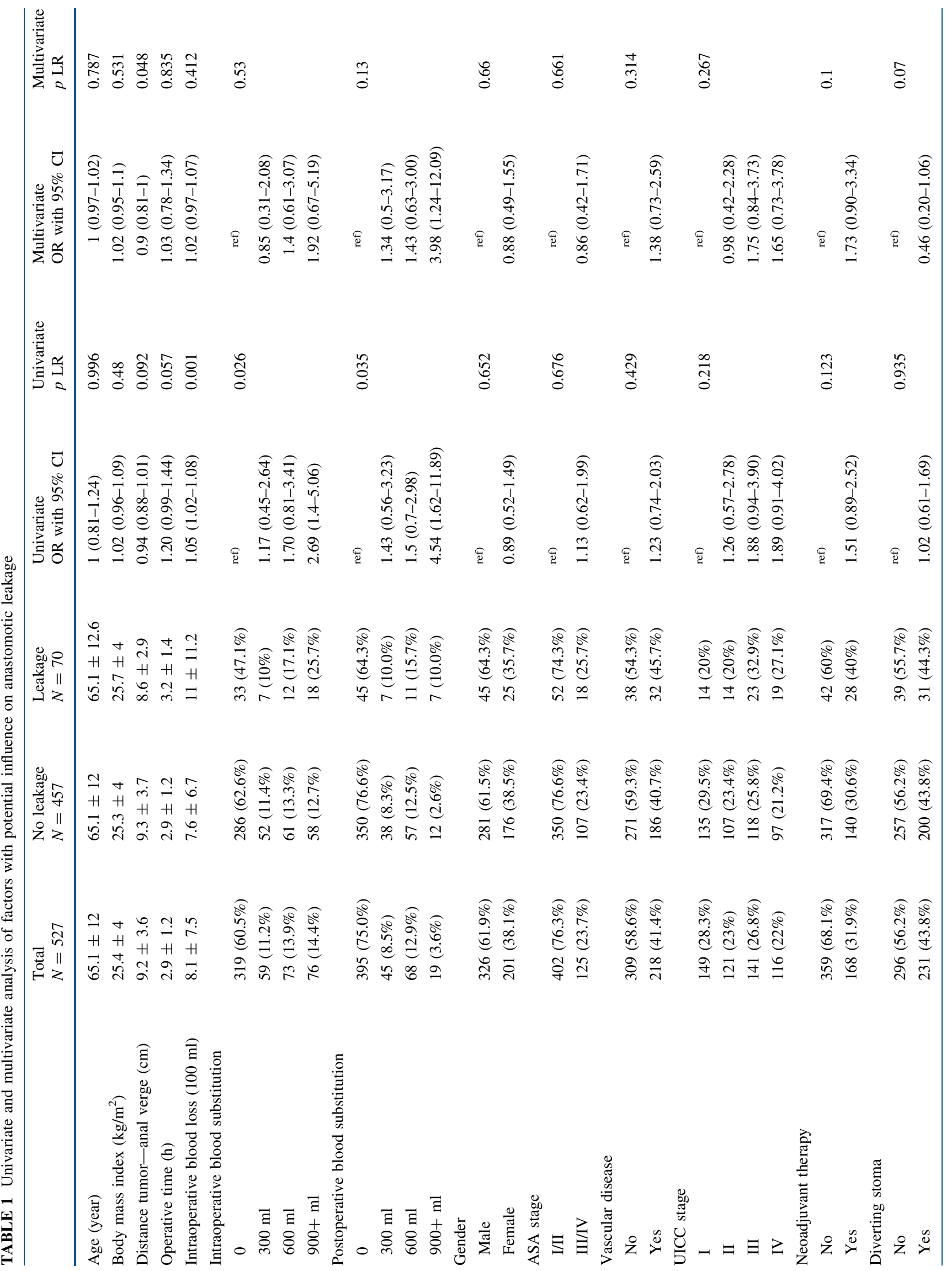


TABLE 2 Backward selection from multivariate full model analysis on anastomotic leakage

\begin{tabular}{lll}
\hline & OR with 95\% CI & $p$ LR \\
\hline Intercept & $0.24(0.08-0.77)$ & - \\
Distance tumor_anal verge $(\mathrm{cm})$ & $0.91(0.82-1)$ & 0.051 \\
Intraoperative blood loss $(100 \mathrm{ml})$ & $1.05(1.02-1.08)$ & 0.003 \\
Postoperative blood substitution & & \\
$0 \mathrm{ml}$ & ref) & 0.085 \\
$300 \mathrm{ml}$ & $1.29(0.49-3.01)$ & \\
$600 \mathrm{ml}$ & $1.37(0.62-2.81)$ & \\
$900+\mathrm{ml}$ & $4.05(1.39-11.03)$ & \\
Neoadjuvant radiotherapy & & \\
No & ref) & \\
Yes & $1.59(0.86-2.91)$ & \\
Diverting stoma & & \\
No & ref) & \\
Yes & $0.5(0.24-1)$ & \\
\hline
\end{tabular}

Backward variable selection from full model according to the AICcriterion

Data are odds ratio (OR) with $95 \%$ confidence interval (CI) and significance level from likelihood ratio test unless otherwise indicated

ref) reference category

tumor height, is shown in Fig. 3. A diverting stoma was associated with a lowered risk, independent of distance.

Neoadjuvant radiotherapy was associated with an increased risk if the tumor was located in the middle third of the rectum, but not in the lower third. When vascular disease was present, an increased risk was identified if the tumor was located in the lower part of the rectum. Intraoperative blood substitution and more advanced (UICC stage III and IV) tumors were associated with an increased risk independent of tumor localisation. Gender did not have a relevant effect.

\section{Bootstrap Analysis}

The bootstrap results performing 3,999 times a backward variable selection procedure in permutated samples from the original data with 527 patients each is shown in Table 3. For example, the distance to the anal verge was selected in 3,001 of 3,999 (75\%) permutated samples. In these 3,001 samples with distance to anal verge selected, the median odds ratio was 0.88 (95\% CI, 0.8-0.94) indicating an increasing risk when the distance between the tumor and the anal verge is shorter. In only one sample $(0 \%)$, the odds ratio was higher than unity, indicating the opposite. In the 3,001 samples where the distance to anal verge was selected, this factor reached significance $(p<0.05)$ in $76.5 \%$. Body mass index was selected in $26.1 \%$. When selected, the odds ratio was higher than unity in $93.5 \%$ with a median odds ratio of 1.07 (95\% CI, 
FIG. 2 LOWESS regression analysis with point wise confidence envelopes and mean leakage rate (degree of polynomials $=1$, degree of smoothing $=0.9$ )

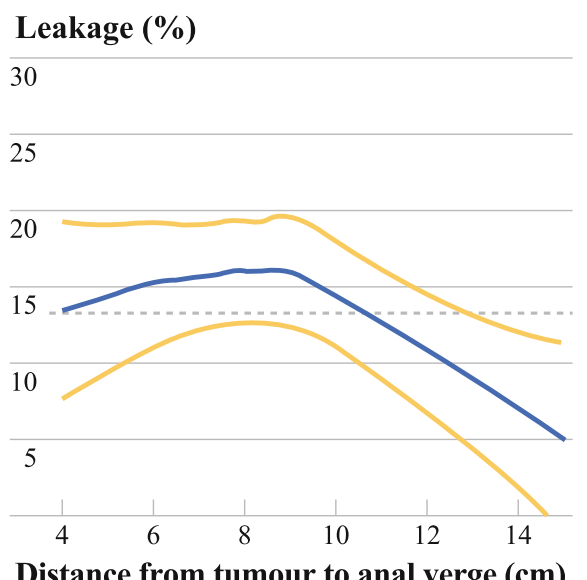

Distance from tumour to anal verge $(\mathrm{cm})$

Leakage (\%)

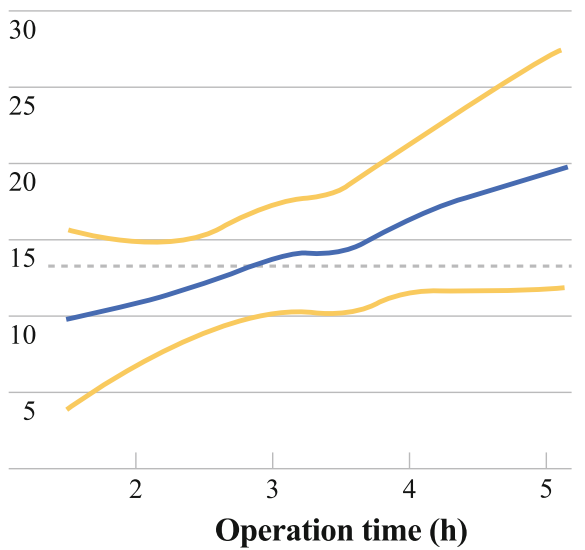

Leakage (\%)

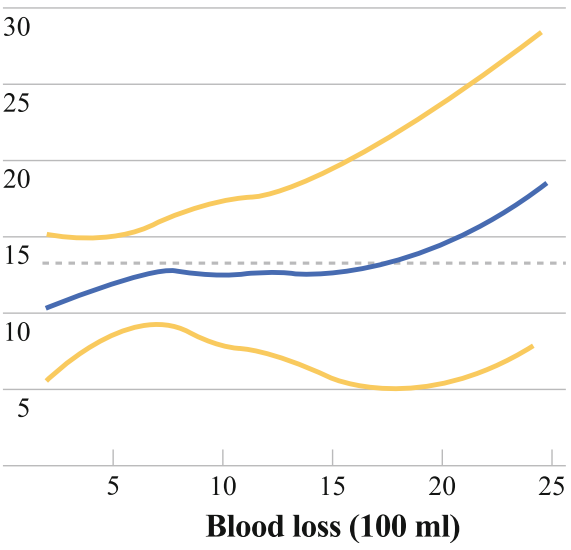

Leakage (\%)

30

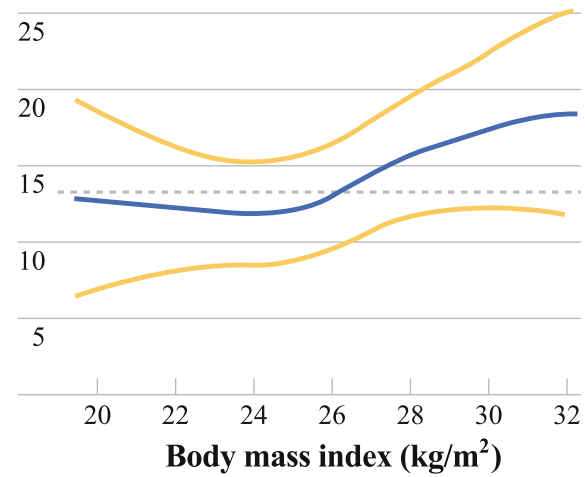

0.94-1.12) and reached significance in 50.2\%. Interpreting the selection rate as an indicator of the importance of a factor, postoperative blood substitution (69.6\%), diverting stoma $(66.9 \%)$, neoadjuvant radiotherapy $(55.9 \%)$, intraoperative blood loss $(53.8 \%)$, and UICC stage $(52.6 \%)$ were important factors, in addition to the distance to the anal verge $(75 \%)$, in developing anastomotic leakage.

Considering the fraction of odd ratios greater than unity as the direction of a factors' influence, postoperative blood substitution of three or more blood bottles (99.4\%), neoadjuvant radiotherapy $(99.1 \%)$, higher intraoperative blood loss $(97.5 \%)$, intraoperative blood substitution of three or more blood bottles $(96.1 \%)$, pre-existing vascular disease (95.5\%), UICC stage III or IV $(95.7 \%$ or $91.5 \%$, respectively), and higher body mass index $(93.5 \%)$ were associated with an increased risk of anastomotic leakage. Similarly, for greater distances to the anal verge $(0 \%)$ and when performing a diverting stoma $(0.5 \%)$, a decreased risk was identified.

\section{DISCUSSION}

To our knowledge, this is the first study to use modern resampling statistical methods, such as bootstrapping, to obtain model-based inferences regarding anastomotic leakage after rectal cancer resection and reconstruction with a colorectostomy. According to the bootstrap and LOWESS regression, anastomotic leakage was associated with intraoperative or postoperative blood substitution with three or more blood units, higher intraoperative blood loss, pre-existing vascular disease, higher body mass index, more advanced (UICC stage III or IV) tumors, neoadjuvant radiotherapy, and shorter tumor height, whereas a diverting stoma was associated with a decreased risk. The bootstrap method demonstrated the variability in variable selection from multivariate analysis, which is a consequence of multiple correlations between potential risk factors, and increased internal validity of the statistical analysis.

Contrary to the bootstrap findings, traditional analysis yielded confusing results, even in a homogeneous sample of 527 patients. Intraoperative blood loss, blood substitution, and distance to the anal verge only partially reached statistical significance in uni- and multivariate regression. The contradictory results are explainable, because reaching statistical significance depends not only on the effect size, but also on statistical power and sample size. Deducing lack of influence from nonsignificant results may be 


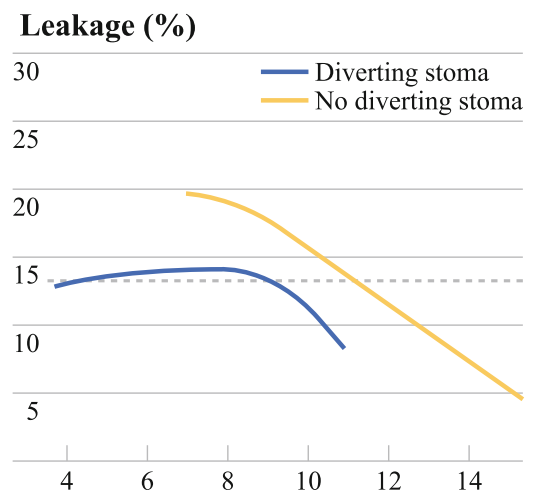

Distance from tumour to anal verge $(\mathrm{cm})$

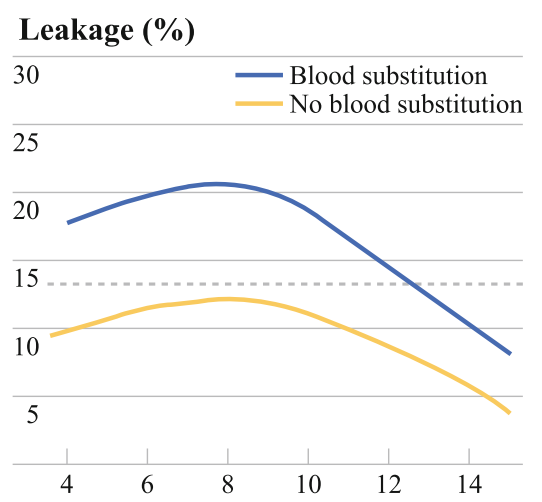

Distance from tumour to anal verge $(\mathrm{cm})$

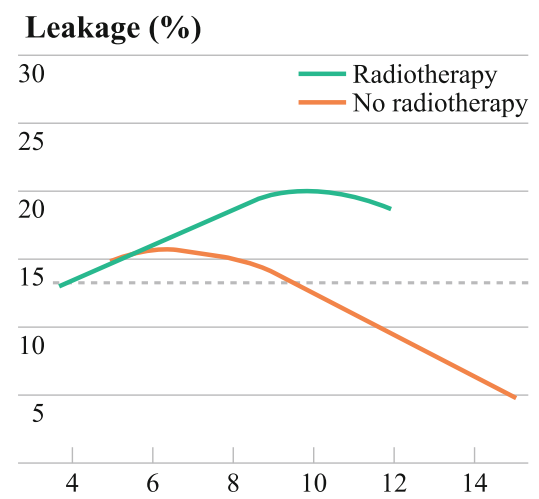

Distance from tumour to anal verge $(\mathrm{cm})$
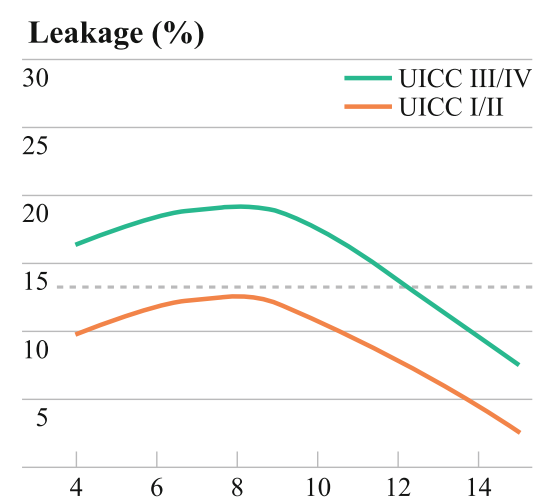

Distance from tumour to anal verge $(\mathrm{cm})$

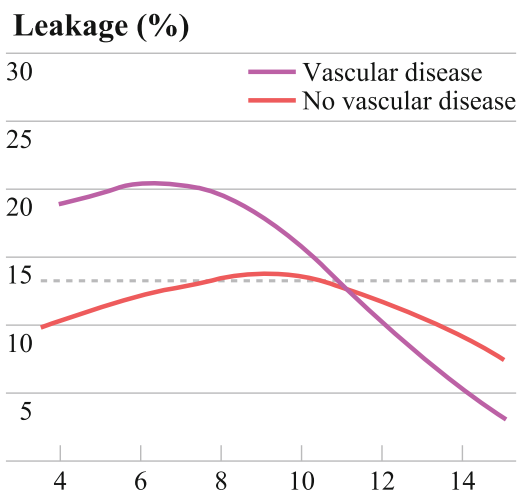

Distance from tumour to anal verge $(\mathrm{cm})$

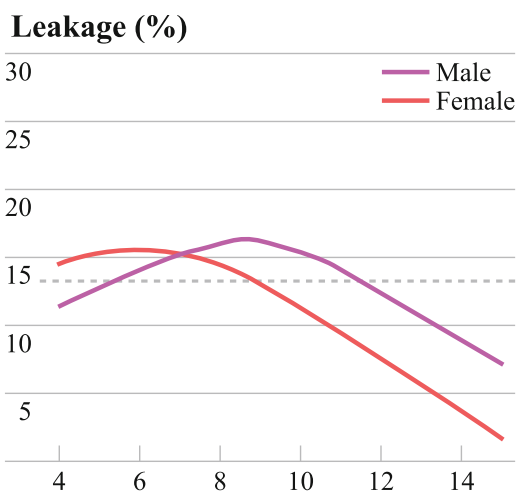

Distance from tumour to anal verge $(\mathrm{cm})$

FIG. 3 LOWESS regression analysis with mean leakage rate for tumor height including covariates (degree of polynomials $=1$, degree of smoothing $=0.9$ )

misleading because absence of evidence is not evidence of absence. $^{19}$

These results are limited to a retrospective, single-center cohort who received surgery between 1991 and 2008 at a tertiary referral hospital. Nevertheless, because all operations were performed or supervised by experienced visceral surgeons and done as highly standardized procedures, the authors consider the cohort to be homogenous without a relevant time effect. However, changes over time in perioperative care and patient characteristics may have biased the results. Furthermore, in the bootstrap results, optimism in estimating odds ratios must be considered.

\section{Diverting Stoma}

Recently, it became evident from RCTs that a diverting stoma may diminish the risk for anastomotic leakage by an odds ratio of approximately $0.29(0.16-0.52){ }^{20,21}$ The crux is that this procedure itself bears a relevant morbidity because $34 \%$ of patients may experience complications related to the ostomy. ${ }^{22}$ Furthermore, surgeon's predictive validity concerning anastomotic leakage is low with a sensitivity of $62 \%$ and a specificity of $52 \%$ in rectal cancer surgery. ${ }^{23}$ In our cohort, $43.8 \%$ of patients received a diverting stoma. In conformity with recent research, our data suggest a decreased risk of leakage in patients with a diverting stoma with an odds ratio of $0.4(0.17-0.61)$. Additionally, it can be assumed that there is a bias toward more diagnosed leakages as a contrast study was routinely performed before closure of a diverting stoma. Furthermore, the bootstrap analysis demonstrated that one third of conventional statistical analysis was unable to identify the role of diverting stoma for preventing anastomotic leakage. Taking into account the morbidity caused by a diverting stoma, promoting the performance of a stoma during rectal cancer surgery seems questionable. Further RCT evaluating leakage after TME should stratify the patients according to a diverting stoma and other risk factors.

\section{Distance to the Anal Verge}

As widely accepted, the distance between the tumor and the anal verge as surrogate for height of anastomosis was proved to be a relevant risk factor for occurrence of anastomotic leakage. ${ }^{3,5,23,24}$ Whereas many studies use the height of anastomosis, the authors, like others, ${ }^{21}$ decided to use the distance between the tumor and the anal verge because it can be estimated more precisely in retrospective 
TABLE 3 Bootstrapping backward variable selection of potential influence factors on anastomotic leakage with a 3,999 times permutated sample containing 527 patients each

\begin{tabular}{|c|c|c|c|c|}
\hline & Selected $[\%]^{\mathrm{a}}$ & $\mathrm{OR}>1[\%]^{\mathrm{b}}$ & Median odds ratio with $95 \% \mathrm{CI}^{\mathrm{c}}$ & $p \operatorname{LR}<0.05[\%]^{\mathrm{d}}$ \\
\hline Age (year) & 19.9 & 24.2 & $0.98(0.96-1.03)$ & 48 \\
\hline Body mass index $\left(\mathrm{kg} / \mathrm{m}^{2}\right)$ & 26.1 & 93.5 & $1.07(0.94-1.12)$ & 50.2 \\
\hline Distance tumor-anal verge $(\mathrm{cm})$ & 75 & 0 & $0.88(0.8-0.94)$ & 76.5 \\
\hline Operative time (h) & 21.1 & 79.4 & $1.27(0.7-1.64)$ & 55.5 \\
\hline Intraoperative blood loss $(100 \mathrm{ml})$ & 53.8 & 97.5 & $1.05(1.02-1.09)$ & 82.2 \\
\hline Intraoperative blood substitution & 40.3 & & & 84.2 \\
\hline 0 & & ref) & ref) & \\
\hline $300 \mathrm{ml}$ & & 38.8 & $0.81(0.17-2.81)$ & \\
\hline $600 \mathrm{ml}$ & & 86.4 & $1.86(0.53-3.97)$ & \\
\hline $900+\mathrm{ml}$ & & 96.1 & $3.04(0.86-6.47)$ & \\
\hline Postoperative blood substitution & 69.6 & & & 84.2 \\
\hline 0 & & ref) & ref) & \\
\hline $300 \mathrm{ml}$ & & 69.6 & $1.38(0.34-3.43)$ & \\
\hline $600 \mathrm{ml}$ & & 80.5 & $1.57(0.50-3.49)$ & \\
\hline $900+\mathrm{ml}$ & & 99.4 & $5.62(1.85-17.48)$ & \\
\hline Gender & 22.6 & & & 43.9 \\
\hline Male & & ref) & ref) & \\
\hline Female & & 15.0 & $0.59(0.36-1.95)$ & \\
\hline ASA stage & 22.1 & & & 42.9 \\
\hline $\mathrm{I} / \mathrm{II}$ & & ref) & ref) & \\
\hline III/IV & & 28.7 & $0.55(0.3-2.35)$ & \\
\hline Vascular disease & 32.3 & & & 54.5 \\
\hline No & & ref) & ref) & \\
\hline Yes & & 95.5 & $1.81(0.59-3.08)$ & \\
\hline UICC stage & 52.6 & & & 73.4 \\
\hline I & & ref) & ref) & \\
\hline II & & 47.0 & $0.96(0.32-2.95)$ & \\
\hline III & & 95.7 & $2.24(0.93-4.71)$ & \\
\hline IV & & 91.5 & $2.13(0.69-4.89)$ & \\
\hline Neoadjuvant therapy & 55.9 & & & 73.7 \\
\hline No & & ref) & ref) & \\
\hline Yes & & 99.1 & $2.15(1.58-4.24)$ & \\
\hline Diverting stoma & 66.9 & & & 75.7 \\
\hline No & & ref) & ref) & \\
\hline Yes & & 0.5 & $0.40(0.17-0.61)$ & \\
\hline Surgeons education & 22.3 & & & 45.4 \\
\hline Visceral surgeon & & ref) & ref) & \\
\hline Board certified & & 16.1 & $0.58(0.36-1.91)$ & \\
\hline Anastomosis & 18.7 & & & 48.1 \\
\hline End to end & & ref) & ref) & \\
\hline End to side/pouch & & 47.9 & $0.58(0.28-3.29)$ & \\
\hline
\end{tabular}

Results of bootstrapping a backward variable selection from a full model according to the AIC-criterion

${ }^{\text {a }}$ How often the factor was selected in the 3,999 analyses

b The fraction of odds ratios greater than unity when the factor was selected

c The bootstrap odds ratio (OR) with $95 \%$ bootstrap confidence interval (CI) when the factor was selected

d The fraction with $p<0.05$ in likelihood ratio tests when the factor was selected

ref) reference category 
analysis compared with the height of anastomosis, because the latter is intraoperatively difficult to measure. Subtracting the distance between the resection margin and the tumor in pathological preparations $(2.9 \pm 2.1 \mathrm{~cm}$ in our cohort) from the distance between the tumor and the anal verge produces the height of the anastomosis. Furthermore, the distance between the tumor and the anal verge is known preoperatively and can be discussed with the patient when deciding whether to perform a diverting stoma.

In this study, the distance to the anal verge was the most often selected factor in the bootstrap analysis with an odds ratio of 0.88 per $\mathrm{cm}$. Additionally, LOWESS regression suggested an increasing risk for shorter distances, although this relationship was limited for distances exceeding $10 \mathrm{~cm}$. The lack of further increased risk for shorter distances may be because a diverting stoma was done in cases with shorter distances. For categorization, a distance between the tumor and the anal verge of $10 \mathrm{~cm}$ and a $7-\mathrm{cm}$ height of the anastomosis might be adequate.

\section{Neoadjuvant Radiotherapy}

Consistent with previously reported series, an increased risk of anastomotic leakage was observed after neoadjuvant radiotherapy in the present study. ${ }^{25-29}$ Besides the tendency toward more leakages in conventional analysis, this factor was selected in $55.9 \%$ of the 3,999 bootstrap analyses, yielding a median odds ratio of 2.15 (1.58-4.24) exceeding unity in $99.1 \%$. Because a recently published interim analysis observed a tendency toward more postoperative complications if surgery was delayed beyond 10 days after the start of short-course radiotherapy, the schedule of radiotherapy also should be considered. ${ }^{30}$ Lack of evidence in other studies ${ }^{6,31}$ may be explained by low statistical power or different courses of radiotherapy.

\section{Blood Loss}

This study identified a strong relationship between intraoperative blood loss and the occurrence of anastomotic leakage. In detail, LOWESS-regression revealed no change in the risk between $700 \mathrm{ml}$ and $1500 \mathrm{ml}$ blood loss, and an increased risk when this amount was exceeded. This finding is consistent with previously published data, although blood loss often was categorized. ${ }^{4,23,24,32}$ Whether blood loss and the associated blood substitution causes leakages through immunological changes or is simply a surrogate for technically difficult operations remains uncertain. Because there was no relevant correlation between the distance to the anal verge and blood loss $(r=-0.162)$, the first explanation seems more likely. Nevertheless, blood loss exceeding $1500 \mathrm{ml}$ should be one of the factors that raise questions for a diverting stoma.

\section{Blood Transfusion}

In agreement with previous reports, the results of the current study identified a dose-dependent association between the risk of leakage and allogeneic blood transfusion, either intra- or postoperatively. ${ }^{5,28,32}$ A systemic inflammatory response with changes in plasma concentrations of inflammatory mediators may explain this relationship. ${ }^{33}$ If three or more blood units were transfused intra- or postoperatively, bootstrap yielded an odd ratio above unity in $99.4 \%$ respectively in $96.1 \%$. Other studies did not differentiate between the timing of transfusions. In the current study, the proportion of patients receiving blood transfusions was relatively high with $40 \%$ occurring intraoperatively and $25 \%$ postoperatively. According to our data, the intraoperative need for three or more blood bottles should raise the question of a diverting stoma. Postoperative transfusions may be an early sign of leakage rather than a cause, but because these were only included before the third postoperative day, transfusion preceded the diagnosis of leakage. Thus, the postoperative need for three or more blood bottles should indicate the need for an active search for anastomotic leakage.

\section{Operation Time}

Risk of leakage and operative time were correlated in the univariate analysis and in the LOWESS regression; however, multivariate and bootstrap analysis denied such a relationship. In contrast to this finding, other studies identified operative time as a relevant risk factor, although it was categorized. ${ }^{5,6,34}$ Other studies identified no relationship. ${ }^{8}$ In further analysis, omitting blood loss from the risk set yielded operative time as a relevant risk factor. Because blood loss and operative time were highly correlated $(r=0.584 ; p<0.001)$, the discrepancy between previous reports and this study seem to be explained by collinearity and differing risk sets. Nevertheless, because operative time is a surrogate marker for leakage, if the operative time exceeds 4 hours, raising the question of a diverting stoma may be wise.

\section{Body Mass Index}

Obesity has been cited as a risk factor for colorectal anastomotic leakage. ${ }^{3,5,35}$ In this study, only a weak association between body mass index and leakage rate was observed, with an increased risk when body mass indexes exceeded $25 \mathrm{~kg} / \mathrm{m}^{2}$. Contradictory results with a lack of influence in the traditional analysis in this and other studies may be interpreted as a power issue, because body mass index was selected in only $26.1 \%$ of the bootstrap analyses. $^{4,6,7}$ 


\section{Vascular Disease}

A relevant association of vascular disease with an increased leakage rate was shown by using resampling statistical methods. Bootstrap yielded an odds ratio greater than unity in $95.5 \%$. This result seems reasonable because the development of anastomotic leakage is hypothesized to depend on ischemia. Furthermore, diminished colorectal tissue oxygenation is predictive for subsequent leakage. Although the effect may be well explained, only a few studies showed such a relationship directly or indirectly, using smoking as a surrogate for vascular disease. ${ }^{32,36}$

\section{UICC Stage}

Patients with advanced tumors (UICC stage III and IV) were more prone to anastomotic leakage with odds ratios greater than unity in $95.7 \%$ and $91.5 \%$ of stage III and stage IV cases, respectively, in the bootstrap analysis. Only a few studies reported a significant correlation between UICC stage and leakage rates. ${ }^{2}$ Other studies described only a tendency. ${ }^{6,8}$ This association may be interpreted as a bias for more distal anastomoses, because larger tumors require more extensive resections, resulting in more shortening of the height of the anastomosis. Nevertheless, if staging yields an UICC stage III rectal cancer, the performance of a diverting stoma could be advocated.

\section{Gender}

In a traditional analysis, gender was not significantly associated with anastomotic leakage. In the bootstrap analysis, gender was selected as a relevant factor in only $22.6 \%$. When selected, the median odds ratio for female sex was 0.59 and less than unity in $85 \%$, indicating a tendency toward decreased risk. Thus, our data do not indicate a strong effect for gender for anastomotic leakage, although the wider pelvis in females could explain such a finding, which was identified by others. ${ }^{2,5,8,31}$

\section{Surgeons Education}

This study yielded a weak association between surgeon's education and the leakage rate in the bootstrap analysis. When the operation was conducted by boardcertified surgeons, the risk was smaller compared with visceral surgeons. All rectal cancer resections are supervised or done by specially trained visceral surgeons. Thus, this weak effect may be interpreted as technically easier cases being more often delegated to less experienced surgeons for teaching purposes. Similar results were presented by Sorensen et al., who showed a significant decrease in leakage rate, with an odds ratio of 0.19 , with trainee surgeons. ${ }^{32}$

\section{Type of Anastomosis, Age, and ASA Classification}

The patient's age, performing the anastomosis end-toend versus end-to-side with and without a pouch, and ASA classification did not show a relevant correlation with the occurrence of anastomotic leakage in any of the analyses. Thus, in this cohort, a relevant effect was not identified.

This study confirmed a decreased risk of leakage after descendorectostomy in rectal cancer surgery after performance of a diverting stoma, and for greater distances between the tumor and the anal verge. An adverse effect of neoadjuvant radiotherapy on leakage rate was observed. Applying statistical resampling methods increased the internal validity of this study, and enabled the identification of intraoperative blood loss, blood substitution, pre-existing vascular disease, and advanced UICC stage as relevant risk factors for anastomotic leakage in one study population.

\section{ANNOTATION}

Applying modern statistical methods may help to end the confusion about risk factors for anastomotic leakage. To accelerate the process, the data analysis provided in this article is mimicked in an exemplary statistical script with artificial random data for usage with the open source $R$ statistical software.

\section{SOURCES OF FINANCIAL SUPPORT None.}

\section{REFERENCES}

1. Nastro P, Beral D, Hartley J, Monson JR. Local excision of rectal cancer: review of literature. Dig Surg. 2005;22:6-15.

2. Ptok $\mathrm{H}$, et al. Impact of anastomotic leakage on oncological outcome after rectal cancer resection. Br J Surg. 2007;94: $1548-54$.

3. Kingham TP, Pachter HL. Colonic anastomotic leak: risk factors, diagnosis, and treatment. J Am Coll Surg. 2009;208:269-78.

4. Jestin P, Pahlman L, Gunnarsson U. Risk factors for anastomotic leakage after rectal cancer surgery: a case-control study. Colorectal Dis. 2008;10:715-21.

5. Makela JT, Kiviniemi H, Laitinen S. Risk factors for anastomotic leakage after left-sided colorectal resection with rectal anastomosis. Dis Colon Rectum. 2003;46:653-60.

6. Vignali A, et al. Factors associated with the occurrence of leaks in stapled rectal anastomoses: a review of 1,014 patients. $J$ Am Coll Surg. 1997;185:105-13.

7. Choi HK, Law WL, Ho JW. Leakage after resection and intraperitoneal anastomosis for colorectal malignancy: analysis of risk factors. Dis Colon Rectum. 2006;49:1719-25.

8. Peeters KC, et al. Risk factors for anastomotic failure after total mesorectal excision of rectal cancer. Br J Surg. 2005;92:211-6.

9. Efron B. Bootstrap methods: another look at the jackknife. Ann Stat. 1979;7:1-26. 
10. Boos DD. Introduction to the bootstrap world. Stat Sci. 2003;18:168-74.

11. Altman DG, Andersen PK. Bootstrap investigation of the stability of a Cox regression model. Stat Med. 1989;8:771-83.

12. Austin PC, Tu JV. Bootstrap methods for developing predictive models. Am Stat. 2004;58:131-7.

13. Cleveland WS, Devlin SJ. Locally-weighted regression: an approach to regression analysis by local fitting. J Am Stat Assoc. 1988;83:596-610.

14. Zaheer S, et al. Surgical treatment of adenocarcinoma of the rectum. Ann Surg. 1998;227:800-11.

15. Knight CD, Griffen FD. An improved technique for low anterior resection of the rectum using the EEA stapler. Surgery. 1980;88:710-4.

16. Dindo D, Demartines N, Clavien PA. Classification of surgical complications: a new proposal with evaluation in a cohort of 6336 patients and results of a survey. Ann Surg. 2004;240: 205-13.

17. Clavien PA, et al. The Clavien-Dindo classification of surgical complications: five-year experience. Ann Surg. 2009;250:187-96.

18. Agresti A, Coull BA. Approximate is better than "exact" for interval estimation of binomial proportions. Am Stat. 1998;52: 119-26.

19. Altman DG, Bland JM. Absence of evidence is not evidence of absence. BMJ. 1995;311:485.

20. Ulrich AB, Seiler C, Rahbari N, Weitz J, Buchler MW. Diverting stoma after low anterior resection: more arguments in favor. Dis Colon Rectum. 2009;52:412-8.

21. Matthiessen P, Hallbook O, Rutegard J, Simert G, Sjodahl R. Defunctioning stoma reduces symptomatic anastomotic leakage after low anterior resection of the rectum for cancer: a randomized multicenter trial. Ann Surg. 2007;246:207-14.

22. Gunnarsson U, Karlbom U, Docker M, Raab Y, Pahlman L. Proctocolectomy and pelvic pouch - is a diverting stoma dangerous for the patient? Colorectal Dis. 2004;6:23-7.

23. Karliczek A, et al. Surgeons lack predictive accuracy for anastomotic leakage in gastrointestinal surgery. Int J Colorectal Dis. 2009;24:569-76.

24. Law WL, Chu KW. Anterior resection for rectal cancer with mesorectal excision: a prospective evaluation of 622 patients. Ann Surg. 2004;240:260-8.
25. Valero G, et al. Neoadjuvant radiation and chemotherapy in rectal cancer does not increase postoperative complications. Int $J$ Colorectal Dis. 2003;18:495-9.

26. Buie WD, MacLean AR, Attard JA, Brasher PM, Chan AK. Neoadjuvant chemoradiation increases the risk of pelvic sepsis after radical excision of rectal cancer. Dis Colon Rectum. 2005;48:1868-74.

27. Folkesson J, Nilsson J, Pahlman L, Glimelius B, Gunnarsson U. The circular stapling device as a risk factor for anastomotic leakage. Colorectal Dis. 2004;6:275-9.

28. Alves A, et al. Factors associated with clinically significant anastomotic leakage after large bowel resection: multivariate analysis of 707 patients. World J Surg. 2002;26:499-502.

29. Camma C, et al. Preoperative radiotherapy for resectable rectal cancer: a meta-analysis. JAMA. 2000;284:1008-15.

30. Pettersson D, et al. Interim analysis of the Stockholm III trial of preoperative radiotherapy regimens for rectal cancer. Br J Surg. 2010;97:580-7.

31. Law WI, Chu KW, Ho JW, Chan CW. Risk factors for anastomotic leakage after low anterior resection with total mesorectal excision. Am J Surg. 2000;179:92-6.

32. Sorensen LT, et al. Smoking and alcohol abuse are major risk factors for anastomotic leakage in colorectal surgery. Br J Surg. 1999;86:927-31.

33. Miki $\mathrm{C}$, et al. Perioperative allogeneic blood transfusion, the related cytokine response and long-term survival after potentially curative resection of colorectal cancer. Clin Oncol ( $R$ Coll Radiol). 2006;18:60-6.

34. Konishi T, Watanabe T, Kishimoto J, Nagawa H. Risk factors for anastomotic leakage after surgery for colorectal cancer: results of prospective surveillance. J Am Coll Surg. 2006;202:439-44.

35. Rullier E, et al. Risk factors for anastomotic leakage after resection of rectal cancer. Br J Surg. 1998;85:355-8.

36. Martel G, et al. Neoadjuvant therapy and anastomotic leak after tumor-specific mesorectal excision for rectal cancer. Dis Colon Rectum. 2008;51:1195-201. 